\title{
A phonological interpretation of two acoustic confusion matrices
}

\author{
D. J. SHAW \\ Darwin College, University of Kent at Canterbury, Canterbury, Kent CT2 7NY, England
}

\begin{abstract}
Multidimensional scaling and principal component analysis methods are used to examine two acoustic confusion matrices. It is suggested that three of the dimensions isolated by means of these techniques can be identified with phonological phenomena which could have contributed to the confusions.
\end{abstract}

In a recent paper in this journal, Morgan (1973) investigated the use of cluster analysis methods to examine two "acoustic confusion matrices." $\mathrm{He}$ shows that these methods provide adequately consistent analyses of the two sets of data and he also makes some suggestions as to the phonological interpretation of these results. It is, in fact, possible to extract considerably more phonological information than Morgan suggests. It is the intention of this paper to emphasize the consistency of the cluster analyses by putting forward further interpretations of underlying phonological patterns common to both sets of results. ${ }^{1}$

A description of the experiments by Conrad (1964) and Hull (1973) is given by Morgan (1973), together with an account of the representation of the data by means of a dissimilarity matrix for the single-link cluster analysis (SLCA) and multidimensional scaling (MDS) programs.

In addition to a phonological assessment of the SLCA clusters, the present paper is particularly concerned to explore further Morgan's use of principal component analysis as a method of projecting MDS configurations with high dimensionality on to planes which can be plotted and inspected visually. The technique of projection on to principal components of MDS configurations has been used by Kendall (1971) and by Sibson (1972). Morgan (1973) also used the technique on acoustic data due to Wickelgren (1965). Further, in an experiment on an artiticial four-dintensional set of data to which a considerable random distortion was added, Morgan wals able to recover all four dimensions of the original data by means of the MDS and principal component analysis methods used on the two acoustic confusion matrices examined here. Therefore, although MDS gives only a monotonic regression of distance on dissimilarity and the principal component analysis works on the actual distances of the MDS contiguration. the combination of these techniques may be expected to yield useful results on these data.

For both sets of data (26 alphabetic stimuli $\mathrm{A}-\mathrm{Z}$ and 35 alphanumeric stimuli $A-Z$ 0-9), a five-dimensional
Table 1

Percentage of Variation for Each Dimension Shown by Principal Component Analysis

\begin{tabular}{|c|c|c|}
\hline \multirow[b]{2}{*}{ Dimension } & \multicolumn{2}{|c|}{ Percentage of Variation } \\
\hline & 35 Items & 26 Items \\
\hline 1 & 37 & 33 \\
\hline 2 & 24 & 28 \\
\hline 3 & 16 & 18 \\
\hline 4 & 14 & 13 \\
\hline 5 & 10 & 8 \\
\hline
\end{tabular}

MDS analysis was performed, using Kruskal's (1964a, b) method. In each case, the program was run several times with different random starts and the contigurations with the lowest stress values $(7.28 \%$ and $12.35 \%$ ) were chosen for input to a five-dimensional principal component analysis program. Table 1 shows the percentage of variation of the five dimensions for both sets of data. Tables 2 and 3 list the complete results of the principal component analyses.

\section{PHONETIC SYMBOLS}

In the analysis of phonetic data, it is important to use a more informative notation than the conventional representation (ABC ... XYZ 012 . . 9) which reveals nothing about the phonetic nature of the stimuli. In Table 4 , the 35 items are presented in a form of phonetic alphabet which will be used in this paper. The representation given here is a generalized one based on standard British English. The notation used is not intended to be rigorous, but merely to represent the data as adequately as seemed necessary with a minimum of special symbols. It is hoped that this gives a satisfactory approximation of the sounds used in the experiments. I have not had access to recordings of the voices actually used. (In Morgan, Chambers. and Morton, 1973, Table 8, a phonetic transcription of the 10 digits spoken in that experiment is provided.) An account of phonetic notations can be found in most introductory texts on 
Table 2

Results of Five-Dimensional Principal Component Analysis for 26 Alphabetic Stimuli

\begin{tabular}{|c|c|c|c|c|c|c|c|c|c|c|}
\hline & \multicolumn{10}{|c|}{ Dimension } \\
\hline & & 1 & & 2 & & 3 & & 4 & & 5 \\
\hline 1 & 2.10 & ef & 2.48 & eks & 1.72 & $\mathrm{~d} \wedge$ blju & 1.76 & $\mathrm{~d} 3 \mathrm{ei}$ & 1.13 & em \\
\hline 2 & 1.99 & eks & 1.51 & ef & 1.37 & vi: & 1.65 & kei & 1.03 & di: \\
\hline 3 & 1.85 & es & 1.43 & kju: & 1.05 & zed & .72 & wai & .94 & en \\
\hline 4 & 1.60 & zed & 1.36 & si: & 1.03 & ou & .67 & si: & .56 & ei \\
\hline 5 & 1.50 & $\mathrm{~d} \wedge$ blju & 1.35 & es & .76 & $\mathrm{~d} Z \mathrm{ei}$ & .62 & zed & .53 & si: \\
\hline 6 & 1.15 & eit $\int$ & 1.34 & eits & .76 & eitf & .44 & pi: & .52 & ef \\
\hline 7 & 1.14 & el & 1.34 & ti: & .76 & kei & .39 & $\mathrm{ti}:$ & .48 & bi: \\
\hline 8 & .90 & ou & 1.03 & pi: & .61 & kju: & .37 & en & .42 & i: \\
\hline 9 & .68 & ei & .68 & zed & .60 & ju: & .33 & em & .41 & $d \wedge b l j u$ \\
\hline 10 & .59 & kei & .42 & $\mathrm{i}:$ & .60 & ei & .31 & $\mathrm{ei}$ & .27 & es \\
\hline 11 & .47 & $a:$ & .12 & $\mathrm{bi}:$ & .41 & bi: & .27 & bi: & .22 & kei \\
\hline 12 & .29 & en & .09 & $\mathrm{di}:$ & .40 & di: & .20 & kju: & .20 & vi: \\
\hline 13 & .16 & $\mathrm{em}$ & -.05 & ju: & .27 & pi: & .12 & ef & .14 & pi: \\
\hline 14 & .01 & $d Z_{e i}$ & -.26 & vi: & .09 & $\mathrm{ti}:$ & .09 & es & .08 & ti: \\
\hline 15 & -.28 & $\mathrm{ai}^{2}$ & -.30 & $\mathrm{~d} 3 \mathrm{i}:$ & .01 & $\mathrm{~d} 3 \mathrm{i}:$ & -.01 & el & -.10 & eks \\
\hline 16 & -.35 & wai & -.54 & $a:$ & -.34 & $\mathrm{eks}$ & -.26 & eits & -.22 & eit! \\
\hline 17 & -1.13 & vi: & -.86 & wai & -.48 & el & -.32 & eks & -.33 & ai \\
\hline 18 & -1.13 & i: & -.91 & ai & -.55 & $\mathrm{i}:$ & -.43 & di: & -.33 & $\mathrm{~d} 3 \mathrm{i}:$ \\
\hline 19 & -1.17 & $\mathrm{di}:$ & -.91 & $\mathrm{~d} \wedge$ blju & -.60 & es & -.44 & vi: & -.45 & el \\
\hline 20 & -1.21 & ti: & -1.04 & $\mathrm{~d} 3 \mathrm{ei}$ & -.68 & ef & -.48 & $\mathrm{~d} 3 \mathrm{i}:$ & -.47 & ju: \\
\hline 21 & -1.21 & ju: & -1.14 & kei & -.80 & si: & -.50 & ou & -.50 & zed \\
\hline 22 & -1.30 & pi: & -1.22 & en & -.86 & en & -.51 & i: & -.51 & ou \\
\hline 23 & -1.44 & kju: & -1.26 & $\mathrm{em}$ & -1.38 & $\mathrm{em}$ & -.72 & $a:$ & -.76 & d3ei \\
\hline 24 & -1.50 & si: & -1.41 & el & -1.43 & wai & -1.27 & ju: & -.99 & $a:$ \\
\hline 25 & -1.58 & bi: & -1.50 & ou & -1.58 & $a:$ & -1.30 & ai & -1.05 & kju: \\
\hline 26 & -2.14 & d3i: & -1.75 & ei & -1.74 & ai & -1.68 & $\mathrm{~d} \wedge \mathrm{blju}$ & -1.22 & wai \\
\hline
\end{tabular}

linguistics (e.g., Gleason, 1961, pp. 254, 315). A very useful description of British English is given in O'Connor (1973).

In the analysis that follows, the term "phoneme" is employed. A phoneme is "a minimum feature of the expression system of a spoken language" (Gleason, 1961 , p. 9) and it is shown enclosed by a pair of slashes, e.g., /i/, as opposed to phonetic distinctions, which are represented by square brackets, e.g., [i]. It is often difficult to decide whether diphthongs should be treated as single phonemes or not. In this paper. they have been considered to be single phonemes: for example, the diphthong / ai/ is a single phoneme combining the sounds $[a]$ and [i], each of which can be a distinct phoneme in other circumstances. The diphthong $/ \mathrm{i}: /$ is a different phoneme from the unlengthened $/ \mathrm{i} /$.

\section{SLCA CLUSTERS}

The clusters formed by Morgan's SLCA analyses (Morgan, 1973, Figures 1-5) inspire confidence on phonological grounds, since both sets of data are similarly treated. In both cases, $W$ forms an isolated cluster of a unique point. This is not surprising. since /dablju/ resembles no other stimulus in the data, being in particular much longer (six phonemes, three syllables).
The major clusters appear to be largely vocalic in nature. In each case, one contains all the $/ \mathrm{i}$ / $/$ and $/ \mathrm{u}: /$ sounds, and there is a clear tendency to group the $/ \mathrm{e} / . / \mathrm{ei} /$, and $/ \mathrm{ai} /$ phonemes in consistent clusters.

Both dendrograms show a distinct /ef//es/ /eks/ cluster where the confusion is obviously due to the pattern $/ \mathrm{e} /$ + fricative. Morgan (1973, p. 22) suggests that the clusters show a tendency for initial phonemes to be masked in recognition, citing the cluster of consonant $+/ \mathrm{i}$ / / and the separation of $/ \mathrm{e} /$ + nasal $(/ \mathrm{en} / \mathrm{and} / \mathrm{em} /$ ) from the cluster of $/ \mathrm{e} /+$ fricative (/ef//es//eks/). The absence of a full set of contrasting pairs in the data (e.g.. there is no /ne/ or $/ \mathrm{me} /$, or /i:p/ /i:t/ or /i:s/) means that this interesting suggestion cannot be fully explored.

Further interesting cases are the OL cluster and the pronunciation of $Z$. The reason for $O L$ forming a separate cluster in the SLCA analysis of the alphanumeric data is probably the common tendency to vocalize the $/ \mathrm{l} /$ of $\mathrm{L}$, creating a diphthong /eu/ which would confuse quite easily with /ou/. This tendency can also be seen in the $B(2)$ cluster in Morgan's Figure 4.

It can be readily verified from the clusters that $\mathrm{Z}$ was pronounced/zed/in the British manner and not /zi:/ as in American English (Morgan. 1973. p. 13). In both sets of results, $Z$ is located with, or close to, the $[\mathrm{e}]$ sounds and distinct from the [i] sounds. 
Table 3

Results of Five-Dimensional Principal Component Analysis for 35 Alphanumeric Stimuli

\begin{tabular}{|c|c|c|c|c|c|c|c|c|c|c|}
\hline & \multicolumn{10}{|c|}{ Dimension } \\
\hline & \multicolumn{2}{|c|}{1} & \multicolumn{2}{|c|}{2} & \multicolumn{2}{|c|}{3} & \multicolumn{2}{|c|}{4} & \multicolumn{2}{|l|}{5} \\
\hline 1 & 2.71 & nain & 1.70 & eit & 2.13 & $\mathrm{~d} \wedge \mathrm{blju}$ & 2.10 & ou & 1.24 & $\mathrm{em}$ \\
\hline 2 & 2.20 & wai & 1.55 & eit $\int$ & 1.71 & es & 1.61 & fว: & 1.10 & ef \\
\hline 3 & 2.01 & $\mathrm{w} \wedge \mathrm{n}$ & 1.46 & zed & 1.21 & ef & 1.12 & sevn & 1.04 & tu: \\
\hline 4 & 2.00 & fว: & 1.44 & kei & 1.15 & eks & .97 & ju: & .98 & en \\
\hline 5 & 1.98 & ai & 1.29 & en & .97 & ju: & .96 & $\mathrm{em}$ & .81 & kju: \\
\hline 6 & 1.89 & faiv & 1.26 & es & .91 & sevn & .86 & $\theta \mathrm{ri}:$ & .73 & es \\
\hline 7 & 1.79 & $a:$ & 1.25 & siks & .89 & siks & .80 & el & .68 & wai \\
\hline 8 & 1.45 & el & 1.25 & ei & .76 & nain & .79 & kju: & .54 & ou \\
\hline 9 & .73 & $\mathrm{~d} \wedge$ blju & 1.19 & ef & .67 & d $3 i:$ & .47 & $\mathrm{~d} Z \mathrm{i}:$ & .48 & eks \\
\hline 10 & .61 & ou & 1.08 & eks & .61 & kju: & .46 & zed & .46 & ai \\
\hline 11 & .59 & kei & .85 & sevn & .44 & wai & .39 & en & .41 & pi: \\
\hline 12 & .56 & dZei & .83 & el & .37 & $w \wedge n$ & .36 & i: & .31 & eitf \\
\hline 13 & .45 & sevn & .80 & $\mathrm{em}$ & .21 & zed & .26 & tu: & .27 & eit \\
\hline 14 & .17 & ei & .57 & ou & .17 & ou & .20 & vi: & .17 & fo: \\
\hline 15 & .16 & eks & .44 & d了 ei & -.01 & ti: & .15 & dZei & .11 & ti: \\
\hline 16 & .04 & en & .06 & nain & -.04 & si: & .10 & $\mathrm{~d} \wedge$ blju & .07 & $\mathrm{ei}$ \\
\hline 17 & -.01 & $\mathrm{em}$ & -.19 & f ว: & -.04 & em & .09 & $w \wedge n$ & .06 & $a:$ \\
\hline 18 & -.02 & zed & -.31 & bi: & -.10 & tu: & .06 & $\mathrm{ei}$ & .05 & ju: \\
\hline 19 & -.08 & ef & -.40 & $\mathrm{i}:$ & -.11 & di: & -.06 & kei & -.05 & vi: \\
\hline 20 & -.24 & es & -.40 & $\mathrm{ai}$ & -.18 & $\mathrm{ai}$ & -.14 & bi: & -.09 & $\theta$ ri: \\
\hline 21 & -.73 & eitf & -.43 & $\theta \mathrm{ri}:$ & -.19 & faiv & -.33 & nain & -.15 & faiv \\
\hline 22 & -.73 & ju: & -.60 & pi: & -.19 & el & -.34 & es & -.16 & $\mathrm{i}:$ \\
\hline 23 & -.75 & kju: & -.61 & $\mathrm{di}:$ & -.24 & $\mathbf{i}:$ & -.35 & ef & -.22 & nain \\
\hline 24 & -.84 & tu: & -.72 & si: & -.28 & en & -.45 & $\mathrm{di}:$ & -.25 & d $3 i:$ \\
\hline 25 & -.90 & eit & -.74 & faiv & -.43 & eit & -.49 & siks & -.28 & $w \wedge n$ \\
\hline 26 & -.91 & si: & -.80 & $\mathrm{~d} \wedge \mathrm{blj} \mathrm{u}$ & -.62 & eitf & -.53 & wai & -.29 & $\mathrm{di}:$ \\
\hline 27 & -.93 & $\theta$ ri: & -.91 & vi: & -.68 & dŽei & -.67 & eit & -.36 & bi: \\
\hline 28 & -1.19 & ti: & -1.06 & tu: & -.74 & bi: & -.67 & pi: & -.36 & kei \\
\hline 29 & -1.24 & siks & -1.14 & dzi: & -.82 & pi: & -.71 & $a:$ & -.58 & el \\
\hline 30 & -1.43 & pi: & -1.16 & ti: & -.87 & vi: & -.80 & si: & -.89 & siks \\
\hline 31 & -1.53 & vi: & -1.39 & $a:$ & -1.00 & $\theta$ ri: & -.92 & eks & -.89 & sevn \\
\hline 32 & -1.74 & bi: & -1.46 & $w \wedge n$ & -1.19 & ei & -1.09 & $\mathrm{ti}:$ & -1.05 & zed \\
\hline 33 & -1.94 & $d 3 i:$ & -1.47 & wai & -1.30 & kei & -1.33 & ai & -1.07 & si: \\
\hline 34 & -1.97 & $\mathrm{i}:$ & -1.57 & kju: & -1.55 & f ว: & -1.42 & eitf & -1.16 & $\mathrm{~d} \wedge \mathrm{blju}$ \\
\hline 35 & -2.17 & di: & -1.67 & ju: & -1.61 & $a:$ & -1.44 & faiv & -1.67 & d Jei \\
\hline
\end{tabular}

\section{MDS DIMENSIONS}

It is encouraging to see that the SLCA clusters lend theniselves to phonological analysis, but it is more important to examine the MDS results, since it was the combined use of SLCA, MDS, and principal component analysis which Morgan set out to investigate. Morgan reported that he had "not been able to identify the MDS dimensions with phonetic features" (p. 21). It will be shown here that some such identification is possible and that both sets of results allow similar identifications.

In the case of the first dimensions, identification is quite straighttorward. For both sets of results, we find at one end of the first axis the cluster which contains the /i:/ and / $u$ :/ diphthongs. Clearly, diphthongization is not the element which identifies them, since the opposite end of the axis shows the diphthong/ai/. The next obvious feature shared by [i] and [u] is that they are both classed as close vowels. Close vowels are pronounced with the tongue raised rather close to the palate; open vowels, e.g. [a], are spoken with the tongue low in the mouth (O'Connor, 1973, p. 50). It can be seen from Figure 1 that the cluster of open vowels is at the opposite end of the axis and that the mid-position [e] vowels occur between the two. The first dimension for the 26 alphabetic stimuli shows the same pattern, though not without some interesting variations. For example, the two items, I and Y, containing the diphthong /ai/ are found shifted

Table 4

A Phonetic Transcription Showing the Pronunciation of the Stimuli

\begin{tabular}{llllll}
\hline A & ei & N & en & 1 & wAn \\
B & bi: & O & ou & 2 & tu: \\
C & si: & P & pi: & 3 & $\theta$ ri: \\
D & di: & Q & kju: & 4 & f $: ~$ \\
E & i: & R & a: & 5 & faiv \\
F & ef & S & es & 6 & siks \\
G & d3i: & T & ti: & 7 & sevn \\
H & eit $\int$ & U & ju: & 8 & eit \\
I & ai & V & vi: & 9 & nain \\
J & dZei & W & d $\wedge$ blju & & \\
K & kei & X & eks & & \\
L & el & Y & wai & & \\
M & em & Z & zed & & \\
\hline
\end{tabular}

Note-Vowel length or diphthongization is shown by two dots “:". The meaning of the other special symbols should be clear from the pronunciation. 




Figure 1. Principal Component Dimensions 1 and 2 for 35 alphanumeric stimuli.

towards the close vowels, presumably because the [i] element of the diphthong was more prominent than in the other experiment; the first dimension here sh uws a contrast between the partially open [e] sounds and the close [i] and [u] sounds.

It seems safe to assume that the most important dimension reflects the degree of openness or closeness of the most prominent vowel sound in each stimulus. This fits in well with our earlier observation that the SLCA clusters seem to have a vocalic basis.

This first dimension also appears to correspond to one of the features or "formants" identified in acoustic experiments on vowel sounds. When the vowels are analyzed on a spectrograph (see $\mathrm{O}^{\prime}$ Connor, 1973. chapter 3 ), it is possible to identify two formants as "the two frequency bands at which there is the maximum concentration of energy." These two formants correlate "quite well with their description in terms of the articulatory dimension of front $\mathrm{v}$. back and open v. close" (Lyons, 1968, p. 110). If the arrangement of the stimuli in our first dimension is compared with the frequency values for the first formant of the relevant English vowels [a table of these values is given by Gimson (1972, p. 98) from work done by J. C. Wells], then it can be seen that there is a good correlation. It is probably reasonable to suggest that the first dimension shown in Figure 1 really represents the acoustic frequency pattern of this tirst formant. and that both patterns are to be interpreted as corresponding to the articulatory dimension of open $v$. close. This first formant pattern was also recognized in an experiment on confusion of digits analyzed by MDS and SLCA (Morgan,



Figure 2. Principal Component Dimensions 1 and 3 for 26 alphabetic stimuli.

Chambers, \& Morton. 1973. p. 381). None of the dimensions analyzed in this paper seem to correspond to the second formant (front v. back).

The second dimension for the 35 alphanumeric stimuli appears to be a prosodic one of vowel/consonant position (i.e., the pattern $\mathrm{V}+\mathrm{Cv}$. $\mathrm{C}+\mathrm{V}$ ). The pattern in this case is not so neatly distributed as in the first dimension (see Figure 1). In addition to items with the two-phoneme pattern, there are data which have only a vowel phoneme (A, E, I, $O, R)$ as well as some which have both initial and final consonant groups $(\mathrm{Z}, 1,5,6,7,9)$. In this latter case, the location of the item on the second axis may give some indication of the relative prominence of the two consonants. For example, the $/ \mathrm{n} /$ in $/ w \wedge n /$ seems to be less strongly articulated than the $/ w /$. There are a few items which are out of place if this dimension represents position. The most obvious case is $/ \mathrm{kei} /$. It is possibly located with the vowel + consonant group because the sound [e] is found almost exclusively in that group. [Morgan points out that $\mathrm{K}$ in the original data showed the worst asymmetry (see Morgan, 1973, p. 15) and that this factor might contribute to the misplacing of $\mathrm{K}$.] The $67 \mathrm{Z}$ group is also out of place, probably because the initial sibilant has been masked. the resultant pattern.then appearing simply as vowel + consonant.

When the two-phoneme stimuli alone are considered, the pattern is much clearer. As has been suggested, some of the other stimuli can be assimilated to a two-phoneme pattern because of attenuation of less prominent consonants. The remaining items (e.g. AEIOR) seem fairly randomly 
distributed along the axis of the second dimension. Contirmation of this identification can be sought from the analysis of the 26 alphabetic stimuli where the third dimension shows this prosodic distinction very clearly. Morgan (1973, p. 23) identified this factor in a MDS and principal component analysis of data from a simple confusion experiment by Wickelgren (1965).

The only other dimension which can be safely identified is Dimension 4 of the 35 alphanumeric confusions, which corresponds to Dimension 2 of the 26 alphabetic stimuli. This dimension contains information about the voicing and unvoicing of consonants (e.g., the pairs /bi:/ /pi:/ and /di:/ $/ \mathrm{ti} / /)$. Connected with this feature in acoustic terms is the contrast of fortis and lenis consonants. In English, voiced consonants are usually also lenis (relatively less strongly articulated); unvoiced consonants are fortis (i.e., relatively strongly articulated). Halle, Hughes, and Radley (1957, p. 107) state that the fortis/lenis distinction is more important in English than the correlated factor of voicing (see also $0^{\circ}$ Connor, 1973, pp. 40, 140). Identification of this factor is particularly clear in Dimension 2 of the 26 stimuli results but can be traced without much difficulty in Dimension 4 of the 35 stimuli results.

If Morgan's suggestion that there is a tendency for initial phonemes to be masked in recognition is correct. then this dimension might be expected to provide evidence of better separation of voiced and unvoiced (or fortis and lenis) consonants in the $\mathrm{V}+\mathrm{C}$ pattern than in $\mathrm{C}+\mathrm{V}$. Unfortunately, there are no two-phoneme stimuli with a final voiced consonant in the data, so this suggestion cannot be followed up. The clear evidence of separation of the only strict pairs, /bi:/ / pi:/ and /di://ti:/, suggests that if initial masking is present it cannot be in any way complete.

\section{CONCLUSION}

Morgan's paper shows the usefulness of SLCA and MDS in analyzing large bodies of data and representing them graphically. It now appears that a graphical representation obtained by plotting the dimensions projected on to the principal component axes is not merely a convenience, but may well provide information about real underlying factors which were previously hidden by the structure of the data.

In the results studied here, three of the five dimensions analyzed lent themselves to explanation. and furthermore the same explanations fitted dimensions from both sets of experimental data. In two calses, the phonological factors involved had also been found in work on further sets of data (see Table 5). These correspondences suggest that the mathematical dimensionality of the analysis does conform in these data with "real" dimensions which can be recognized as phonologically reasonable.
Table 5

Identification of the Principal Component Dimensions in Both Sets of Data

\begin{tabular}{lcccc}
\hline & \multicolumn{3}{c}{ Dimension in } & \\
& $\begin{array}{c}35- \\
\text { Item }\end{array}$ & Item & Also Identi- \\
Identification & Data & Data & fied by \\
\hline Open vs. Closed Vowels & 1 & 1 & Morgan et al. \\
V + C vs. C + V & 2 & 3 & Morgan* \\
Fortis vs. Lenis Consonants & 4 & 2 & \\
\hline
\end{tabular}

*In Wickelgren's data

If other experiments in acoustic confusion are to be designed in order to investigate the phonological factors underlying the confusions, it would be desirable to choose stimuli which provide a better distribution of potential factors than is the case in the data examined here. The set A-Z 1-9 contains only 10 out of a possible 20 or so vowel phonemes, being in particular poor in open vowels; about three quarters of the consonant phonemes are present but a number are represented once only. For example, pairs containing precise contrasts of front and back vowels, open and close vowels, voiced and unvoiced consonants, and prosodic pairs (e.g., /bi:/ contrasted with $/ \mathrm{i}: \mathrm{b} /$, /es/ with $/ \mathrm{se} /$, etc.) would need to be included in the stimuli, at the expense, no doubt, of complicating the task of the subjects in noting the sounds which they thought they heard.

\section{REFERENCES}

Conrad, R. Acoustic confusions in immediate memory. British Journal of Psychology, 1964, 55, 75-84.

Grmson, A. C. An introduction to the pronunciation of English (2nd ed.). London: Arnold, 1972.

GLEASON, H. A. An introduction to descriptive linguistics (rev. ed.). New York: Holt, Rinehart and Winston, 1961.

Halle, M., Hughes, G. W., \& Radley, J. P. A. Acoustic properties of stop consonants. Joumal of the Acoustical Society of America, 1957, 29, 107-116.

HuLL, A. J. A letter-digit listening matrix. British Journal of Psychology, 1973, 64, 579-585.

Kendall, D. G. Construction of maps from "odd bits of information." Nature, 1971, 231, 158-159.

KrusKal, J. B. Multidimensional scaling by optimizing goodness of fit to a nonmetric hypothesis. Psychometrika, 1964, 29, 1-27. (a)

KRUSKAL, J. B. Nonmetric multidimensional scaling: A numerical method. Psychometrika, 1964, 29, 115-129. (b)

Lyons, J. Introduction to theoretical linguistics. Cambridge: University Press, 1968.

Morgan, B. J. T. Cluster analyses of two acoustic confusion matrices. Perception \& Psychophysics, 1973, 13, 13-24.

Morgan, B. J. T., Champers, S. M., \& Morton, J. Acoustic confusion of digits in memory and recognition. Perception \& Psychophysics, 1973, 14, 375-383.

O'Connor, J. D. Phonetics. Harmondsworth: Penguin Books, 1973.

Sibson, R. Order invariant methods for data analysis. Journal of the Royal Statistical Society, B, 1972, 34, 311-349. 
WICKelgren, W. A. Similarity and intrusions in short-term memory for consonant-vowel digrams. Quarterly Journal of Experimental Psychology, 1965, 17, 241-246.

\section{NOTE}

1. I should like to thank Dr. Morgan for drawing my attention to the work contained in his paper and for providing additional results from his MDS analyses together with much help and encouragement.
(Received for publication September 5, 1973; revision accepted January 31, 1975.) 